This item was submitted to Loughborough's Research Repository by the author.

Items in Figshare are protected by copyright, with all rights reserved, unless otherwise indicated.

\title{
Cognitive and neuromuscular robotic rehabilitation framework
}

PLEASE CITE THE PUBLISHED VERSION

http://dx.doi.org/10.1007/978-3-319-46562-3_21

\section{PUBLISHER}

Springer

\section{VERSION}

AM (Accepted Manuscript)

\section{PUBLISHER STATEMENT}

This work is made available according to the conditions of the Creative Commons Attribution-NonCommercialNoDerivatives 4.0 International (CC BY-NC-ND 4.0) licence. Full details of this licence are available at: https://creativecommons.org/licenses/by-nc-nd/4.0/

\section{LICENCE}

CC BY-NC-ND 4.0

\section{REPOSITORY RECORD}

Badii, Atta, Maryam Karimi, and Qinggang Meng. 2019. "Cognitive and Neuromuscular Robotic Rehabilitation Framework". figshare. https://hdl.handle.net/2134/24516. 


\title{
Cognitive and Neuromuscular Robotic Rehabilitation Framework
}

\author{
Atta Badii, Maryam Karimi, Qinggang Meng
}

\begin{abstract}
This paper presents a cognitive and neuromuscular robotic rehabilitation framework to support enhanced control of arm movements for humans with muscular control impairment, typically with some level of memory deficiency due to, for example, suffering from a stroke. It describes the design, development and integration of the framework architecture as well as a Baxter robot based demonstration platform. Three key elements of the proposed framework (rehabilitation module, workspace and rehabilitation scenarios) have been described in detail. In the rehabilitation sessions, the users and the robot are asked to work together to place cubes so as to form a predefined shape. The robot and the user hold the same object in order to move it to a particular destination according to a rehabilitation scenario. If the robot detects a force from the user directed in the wrong direction during the navigation then it resists and corrects the movement in order to assist the user towards the right direction. The assistive support scenarios were designed to evaluate the achieved enhancement of precision, efficiency and dexterity of arm movements. The proposed rehabilitation framework provides a modular, automated and opensource platform for researchers and practitioners in neuromuscular rehabilitation applications.
\end{abstract}

\section{Introduction}

Neurological damages caused by accidents, illnesses and birth defects can cause considerable problems to the muscular and neurological function of individuals. These accidents may cause short term physiological or neurological damage which may be treated using physiotherapy and appropriate treatment offered by health ser-

Atta Badii

University of Reading, Reading, UK, e-mail: atta.badii@ reading.ac.uk

Qinggang Meng

Loughborough University, Loughborough, UK, e-mail: q.meng@ lboro.ac.uk 
vices. The nature of such injuries requires continuous attention from the practitioners in order to treat a muscularly impaired individual taking into account the changes in the condition during the period of treatment.

Furthermore, some accidents may cause mid to long term neurological disorders that require a greater level of attention compared to the short term injuries. Neurological injuries are time critical when it comes to rehabilitation and treatment, i.e. if they are not treated in time they can become permanent. The neurological damage may affect any parts of the human body and consequently a patients daily routine, for instance they might not be able to move, feed themselves or move their legs and arms as intended. Some of these disabilities caused by nerve damage are reversible by treatment and special physiotherapies at the right time before they become permanent, this kind of treatment can be a good opportunity for the nerves or muscles to recover.

There is a significant body of clinical experience of recovery trajectories of post stroke patients with limited motor movement and impaired memory who require training and rehabilitation in order to regain their personal capabilities [2, 3]. However, facilitating such training and rehabilitation programmes would require prolonged and intensive physiotherapy sessions and/or care robots which are very resource intensive. Therefore any advances that may lead to efficiency gains in provision of personalised assistive support and training for such patients can generate multiple societal benefits.

The above considerations and the current limitations arising from lack of interoperability and vendor lock-in within robotics and smart home technologies as well as the need for integration of bespoke training have served to motivate work in the area of research focus selected for this study. The overall aim of this study is to enable muscular impairment individuals to evaluate and enhance dexterity and efficiency of their movements by developing a cognitive and neuromuscular robotic rehabilitation framework.

\section{Related Work}

\section{Robotic rehabilitation}

Robotic rehabilitation for upper limbs aids the development of arm strength for individual motor movements. Robotic technologies usually attempt to leverage or enhance neuroplasticity principles through the improvement of movement quality, increasing task repetition and task intensity [2]. Many doctors consider this form of treatment as the future for therapy, particularly in regard to major injuries and severe stroke symptoms.

The current robotic technologies can be used for children suffering from cerebral palsy or those who have undergone orthopaedic surgery. The rehabilitative robots for upper arm rehabilitation act as augmenting devices. Therefore doctors use them as therapy aids rather than assistive devices. The procedure for using these tools 
is non-invasive, and many patients consider the devices to have the sensitivity of a human therapist [2].

Rehabilitation robots used for upper limbs practice number of different techniques during the therapy, these are:

Active Constrained Physical Exercise: The active constrained physical movement of robotic rehabilitation of the upper limb involves the patient's arm moving with an opposing force if the patient's arm attempts to move outside its set physical parameters [2].

Active assisted physical exercise: The active assisted physical exercise, the client, moves their hand in a pathway previously determined by the doctor or therapist without any force opposing or pushing against the arm.

Adaptive exercise: The adaptive physical exercise technique adapted for rehabilitation includes regular exercises of which the robot has no prior knowledge [8]. The standard devices associated with the adaptive physical exercise include MIME and Bi Manu Track.

Passive physical activity: Passive physical exercises despite their name, form part of the robotic rehabilitation of the upper limbs. The rehabilitation requires patients to put in more effort during the session in order to complete the exercise required by the doctor, this technique does not put any force on the limb during the session [6].

Active resistive physical exercise: This exercise, on the other hand, involves an arm movement with directional opposing force. Several robots exist that adapt this technique, including the MIME, Bi-Manu-Track, and MIT Manus.

One of the key benefits of using robot rehabilitation is that the patient can repeat the exercise numerous times over the course of a session or several sessions without any interruption. This type of rehabilitation can also train and enhance the individuals memory and increase the productivity of the patient [7]. The rehabilitation devices are unable to fully understand the patients needs in comparison with an experienced therapist. However, developers in Israel, Japan, Russia, USA and France are designing robots with advanced machine learning algorithms to address this limitation. These robots include the InMotion ARM [10], MITManus of Canada [4] and EskoBionics of Spain/USA [1].

The MIT-MANUS is a proven rehabilitative robotic device offering continuous and customised therapy sessions to patients using a sophisticated control system. The control system permits the device to change the amount of help provided to the patient by analysing the timing of the arm movement and the patient's exact speed of movement [7].

The benefit of this method is an efficient treatment program that negates the need for a human therapist. The MIT Manus system built in America is also extremely effective in improving muscle tone and decreasing spasticity in the targeted upper arm section. It has a unique setting that enables various spatial orientations in the vertical and horizontal planes and an antigravity feature for improving shoulder function.

The InMotionARM or InMotionTechnology robotic rehabilitative device is an American robotic device that senses the force exerted by the patient and assists 
the individual as required. It can continuously adapt to the ability of the patient, which enables the physical therapist to deliver the right number of intensive sensorimotor movements including shoulder retraction/protraction, shoulder external rotation/internal rotation and shoulder extension/flexion. The EskoBionics system, a joint Spanish, and American endeavour uses a powered wearable exoskeleton robot. The system enhances mobility, endurance, and strength. The intelligent version for upper extremities called the AMADEO offers users several types of training strategies such as haptic training, assistive and passive exercises. The patient uses bilateral upper arm therapy and assistive force to improve the shoulder stabilisation, arm movement smoothness, and arm resistive ability [8].

The results of the projects suggest that more people performing rehabilitation using these robots managed to regain the ability to perform simple tasks around their home. The elderly have particularly benefited by restoring mobility that they lost through age related illnesses. It can be established that the results indicate less time spent in rehabilitation, less need for physical therapists and reduced hospital costs. The studies have also led to the discovery of new programming techniques that can refine the movement of the robotic devices and, therefore, an increase in research and development activities. Finally, the project has enabled doctors and researchers to gain a better understanding of how the human muscle and nerves work together in addition to how these interactions can be modelled using Robotic platforms.

Memory and movement The repetitive motor movement can enhance muscular capability of an individual over time, rehabilitation clinics design appropriate training scenarios over the short or long term in order to transfer movement to the short term muscle memory of the patient. In some cases, lack of movement by the individual can negatively affect the memory [5]. The research studies conducted suggest that exercise and regular movement can improve the memory of an individual. A person with minor neuromuscular injuries often suffers from minor memory loss as well. Therefore, the rehabilitation sessions designed for an individual should not only focus on physical movement, but should also focus on simple memory related exercises. There is sufficient evidence presented in the book written by Eric Jensen named Teaching with Brain in Mind [5] which strongly suggests a strong link between movement and learning, that memory is incorporated as the key part of learning. This book proposes that rehabilitation techniques currently adapted for muscle rehabilitation enhance not only the muscular capability of the patient but also contribute towards the memory improvement of the patient throughout the sessions. Therefore, these invaluable findings should be taken into consideration for effective and suitable design of future rehabilitation frameworks.

\section{Robotic Rehabilitation Framework}

In this study, Baxter [9] is used as the hardware platform in the rehabilitation framework. Baxter as shown in figure 1 is an industrial robot, designed and built by a 
Boston-based company named Rethink Robotics. The robot provides different sensors, cameras and high-tech software which enable industrial and academic institutions to integrate it into their applications.

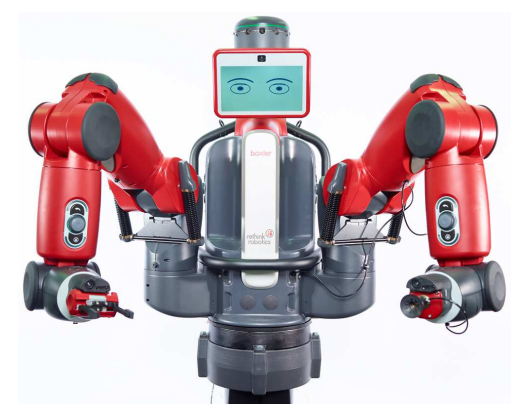

Fig. 1: Baxter robot used in the experiment [9]

In the following, the three key elements of the rehabilitation framework will be described: rehabilitation module; workspace; rehabilitation scenarios.

The Rehabilitation Module was designed to undertake co-ordination of the controllers and make decisions during the rehabilitation sessions. This module receives Arm and Visual information in order to assist the participants during each session. The resistive path correction, object pick up and object placement are three of the main co-ordination tasks managed by this module. Figure 2 shows the overall operation of the rehabilitation module.

Rehabilitation Workspace Figure 3 demonstrates the robot workspace designed for this project which provides a convenient and structured area for the rehabilitation scenarios. There is a designated area to place interacting objects (cubes) and a target grid where the cubes need to be placed during the session. This arrangement allows the participant to sit comfortably in front of Baxter at a safe distance away from the arms while completing the rehabilitation tasks as instructed. The targets on the grid each have a number associated to them; this number will be constant throughout the sessions. The Visual Controller will find and number these targets upon calibration. Figure 3(a) shows the number for each target whereas Figure 3(b) shows the Rehabilitation Workspace. The Rehabilitation Module will use these identifiers to construct specific path plans for targets during rehabilitation sessions.

A number of training scenarios are designed to assist the users with limited motor movement and concentration, the workspace demonstrated in the previous section supports the practical application of the training scenarios.

Before each training scenario the interaction objects will be placed on the side of the targets grid as shown in figure 10. During the session the participant will be instructed to place each cube in the designated locations on the grid. Six different levels or scenarios were designed to demonstrate the functionality of the rehabilitation framework. These include using cubes to create a square in the middle of the 


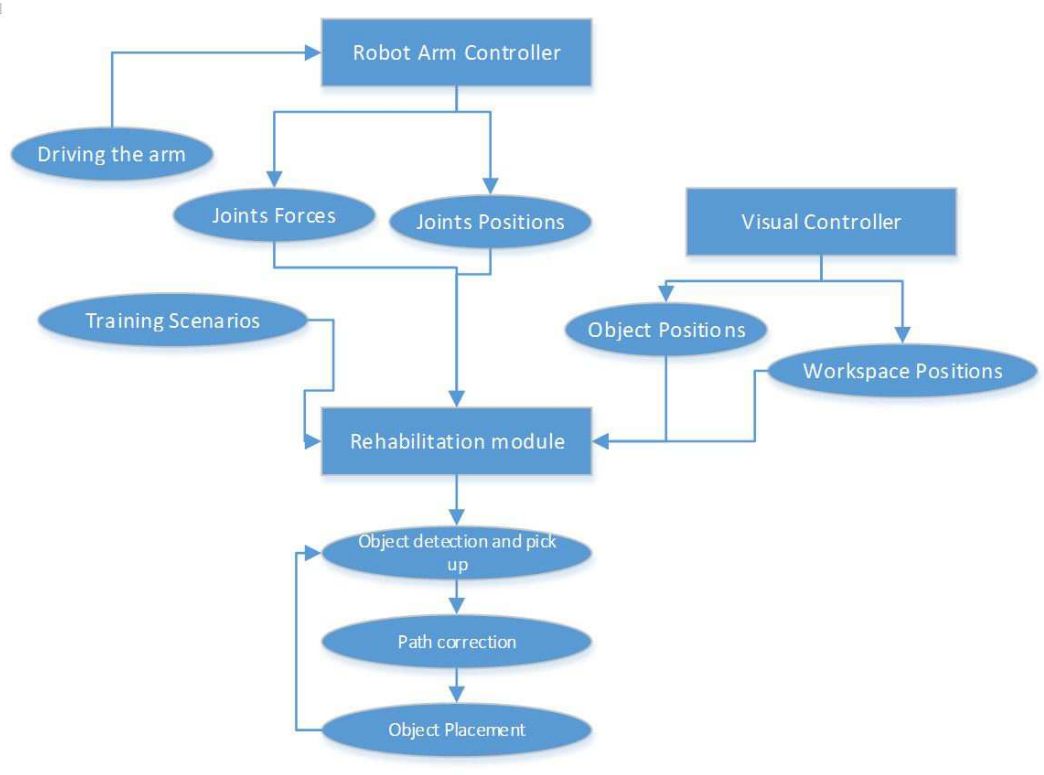

Fig. 2: Rehabilitation operational flow

\begin{tabular}{|c|c|c|c|}
\hline 15 & 14 & 13 & 12 \\
\hline 11 & 10 & 9 & 8 \\
\hline 7 & 6 & 5 & 4 \\
\hline 3 & 2 & 1 & 0 \\
\hline
\end{tabular}

(a) Numbered targeted cells on the workspace

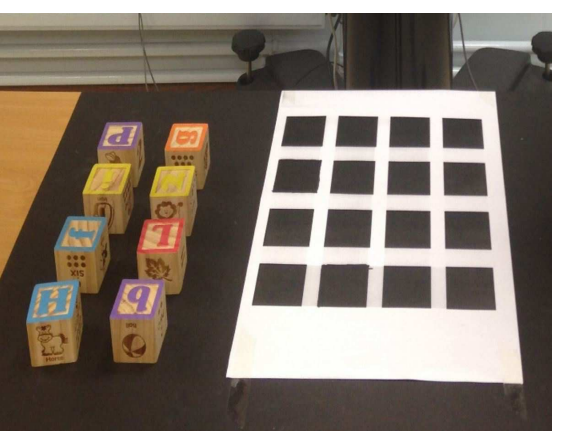

(b) The experimental workspace

Fig. 3: The rehabilitation workspace 
workspace; create a horizontal line; create a vertical line; place four cubes on the corners; place cubes in a Sinusoidal style; and place cubes in a random shape.

During the training, Baxter will pick up the cube for the participant at the beginning of the session. The user will hold one side of the cube while Baxter is holding the other side. The participants moves the cube towards the location desired. If Baxter detects a force directed in the wrong direction from the user during the navigation or guiding process then it resists and corrects the movement in order to assist the user towards the right direction using force applied to the Baxters gripper.

Once the participant is within close proximity of the target, Baxter will slowly help to place the cube and will automatically locate and pick up the next cube for the user. If all the cubes have been collected, Baxter will analyse the current state of the board and will automatically progress to the next level.

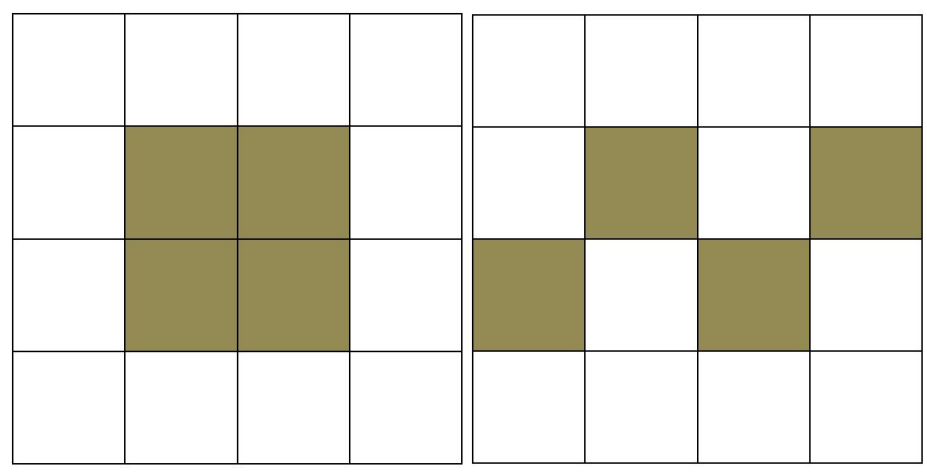

(a) Creating a square in the middle

(b) Placing cubes in sinusoidal pattern

Fig. 4: Examples of training scenarios

\section{Testing and evaluation}

The testing and evaluation of the designed framework was undertaken in order to validate the architecture design and operation of the system. The testing of the framework was conducted in two stages:

- Architecture Testing: The reliability and accuracy of the robot arm and visual controller were tested as part of this stage.

- Operation evaluation: The effectivity of rehabilitation module was evaluated in order to test the operational behaviour of the system during training sessions.

For evaluation 22 tests have been successfully conducted to evaluate each module's functionality in the system, including the robot's visual module to detect the 
workspace accurately; the module of robot loading a different configuration file before each individual training session; robot arm position and force controller; and object detection. One of the key functionalities of the platform is to transform the position from visual space to real world robot position. This feature was tested by measuring the real world position of an object detected, comparing the measured position reported by the visual module. Table 1 shows the results recorded for three different objects in the experiments.

Table 1: Some results of transformation from visual information to real world position

\begin{tabular}{|c|c|c|c|}
\hline Object & Actual position & Reported position & Overall error \\
1 & $0.6,-0.35$ & $0.5913,-0.3392$ & 0.0021 \\
\hline 2 & $0.59,-0.45$ & $0.5821,-0.4391$ & 0.003 \\
\hline 3 & $0.51,-0.36$ & $0.4981,-0.3461$ & 0.002 \\
\hline Average & & & 0.002 \\
\hline
\end{tabular}

The operational capabilities of the rehabilitation module during rehabilitation sessions was tested by placing four cubes in the object area of the workspace, the robot was commanded to begin the rehabilitation session as shown in Figure 5.

The operational behaviour of the robot was recorded for the assessment of using the following testing criteria:

- Detecting and Picking the interaction object: The Baxter robot should be able to successfully find and pick up all four cubes from the table.

- Path Correction and impedance: the robot should be able to effectively provide assistive resistance when an incorrect path is taken. The robot should be able to provide live path correction feedback during the session by applying directional force to resist incorrect movement towards an incorrect direction, and to guide the user towards the right direction.

- Placing: The robot should be able to correctly place the cube in a desired location.

\subsection{Discussions}

The position control and force processing capability of the robot arm controller were also tested during this testing and evaluation stage. The test results confirmed the accuracy of the position controller with an achieved error rate of up to $6 \mathrm{~mm}$ per trial and the force processing functionality showing $83 \%$ accuracy. The data collected about the arm controller satisfies the requirement of the framework for arm related tasks.

The accuracy rate represents distance in millimetre accuracy as average positional error between actual and reported positions on both axis (X and $\mathrm{Y})$, the over- 


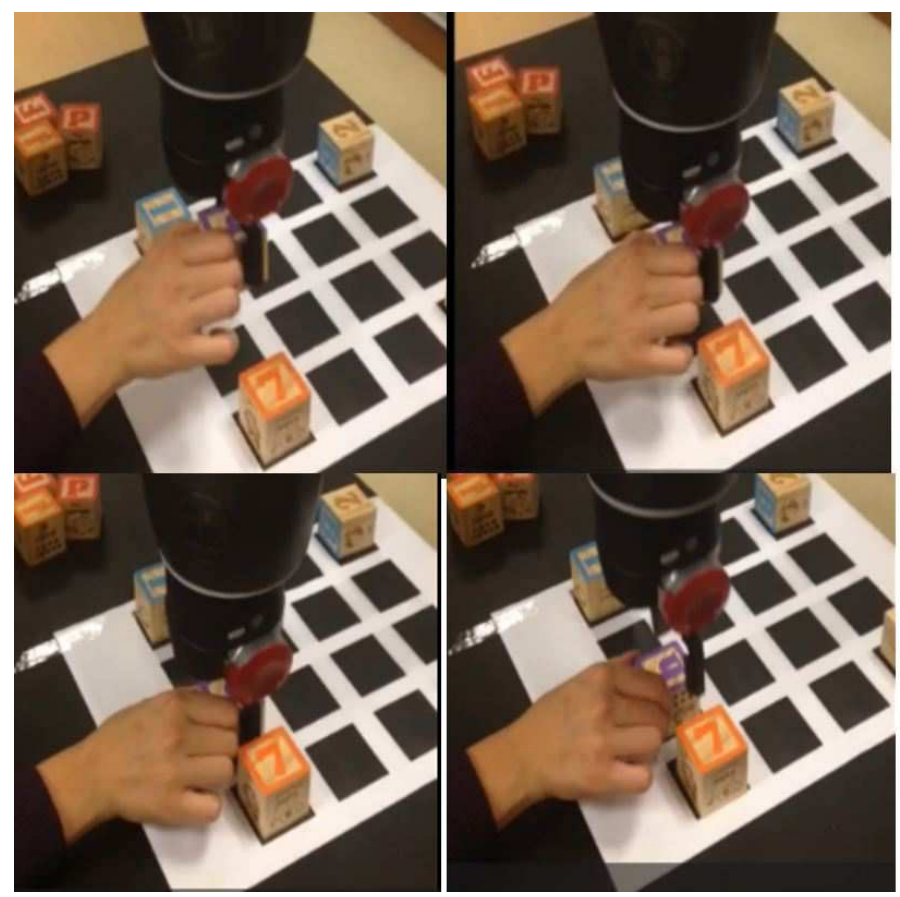

Fig. 5: Object placement evaluation

all result is average of the error for all three objects which amounts to 0.00236 i.e. 0.002 as shown in the tabulated results.

This performance can be significantly improved by integrating adaptive light filtering algorithms to compensate for noise introduced by light in the environment. The information used for calculating this number includes Detected Target Grid and Measured Target Positions.

One of the other architectural test criteria for this paper was to assess the robot capability to correctly load and process the configuration files used during the rehabilitation session. The configuration files were used to provide useful information about the workspace and training scenarios. The results collected during the testing stage provided sufficient evidence to confirm the configuration processing capability of the framework.

The Visual controller designed for the robot met two primary requirements of the project. It was able to accurately detect and locate interacting objects in the workspace; additionally it was able to transform visual information into a real world position with an average error rate of $2 \mathrm{~mm}$.

The operational performance of the robot was evaluated by conducting a number of observations on the behaviour of the robot during the rehabilitation session. The evaluations undertaken confirmed successful implementation of the framework 
to meet the desired objectives of the project. The observed behaviour of the robot demonstrated the robot capability for successful completion of:

1. Object detection, collection and placement

2. Effective Path correction and training via joint resistance

The integrated rehabilitation framework provided an effective and usable solution for two research problems identified in the study. The first problem was the unavailability of open source platforms for hospitals and clinics for the purpose of rehabilitation. The Baxter robot integrated with the Robot Operating System provided a free and open source platform which is a suitable solution for this problem. The second identified problem was a lack of modularity in current rehabilitation frameworks; the designed framework aimed to provide a modular platform enabling integration with additional sensors and devices in addition to flexibility in designing rehabilitation sessions.

\section{Conclusions and future work}

This paper outlines the methodology adopted to design a suitable rehabilitation framework to support enhanced control of arm movement for users with impaired muscular control. The rehabilitation framework provides a modular, automated and open-source platform for researchers and practitioners in neuromuscular rehabilitation applications. The modularity of the framework supports experimentation and adaptation as it offers extensive and flexible development options enabling developers to integrate extra functionalities and features into the system, without being concerned about compatibility issues related to the new development environment. The automation of the platform enables operators to focus on the safety and guidance of the participants instead of considering the operational behaviour of the robot. Most importantly, muscularly impaired individuals can benefit from such a framework to overcome their weaknesses and enhance their motor movement as well as their concentration. In the future, broader human trials will be conducted to validate the framework further. Also, Baxter provides limited force capability for the purpose of rehabilitation. A more powerful resistive robotic arm can be used to improve such capability.

\section{References}

1. E. Bionics. Ekso bionics - an exoskeleton bionic suit or a wearable robot that helps people walk again, ekso bionics. available: http://intl.eksobionics.com/. Accessed July 2015.

2. R Colombo, S Balasubramanian, I Sterpi, and E Burdet. Robotic assessment of upper limb motor function after stroke. American journal of physical medicine and rehabilitation Association of Academic Physiatrists, 91:459- 471, 2012.

3. C Coste and H Kooij. Assistive and rehabiliation robotics. Springer Paladyn Journal, 2:175$188,2013$. 
4. N Hogan, H. I. Krebs, J. Charnnarong, P. Srikrishna, and A. Sharon. Mit-manus: a workstation for manual therapy and training. 1992.

5. Eric Jensen. Chapter 4. Movement and Learning. 2005.

6. H. I. Krebs and et al. Rehabilitation robotics: Performance-based progressive robot-assisted therapy. Automatic Robots, 15:312, 2012.

7. H. I. Krebs and et al. Rehabilitation robotics: Pilot trial of a spatial extension for mit. Manus. Journal of NeuroEngineering and Rehabilitation, 1:233, 2014.

8. L. Marchal-Crespo and D Reinkensmeyer. Review of control strategies for robotic movement training after neurologic injury. Journal of NeuroEngineering and rehabilitation, 6:188-196, 2015.

9. Rethink Robotics. http://www.rethinkrobotics.com/baxter/. Accessed July 2015.

10. I. M. Technologies. Inmotion arm the new standard of care. interactive motion technologies. available: http://interactive-motion.com/inmotion-arm-the-new-standard-of-care. Accessed Aug 2015. 